\title{
LAS EMPRESAS FANTASMAS EN ECUADOR: CARACTERIZACIÓN, SOCIOS Y EMPRESAS RELACIONADAS
}

\section{THE GHOST COMPANIES IN ECUADOR: CHARACTERIZATION, PARTNERS AND RELATED COMPANIES}

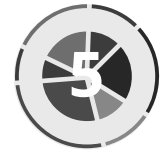

Recibido: 20/04/201

Aceptado: 05/06/2017

\author{
Mayra A. Calderón \\ alexandra.839@ hotmail.com ${ }^{1}$ \\ Jorge L. García \\ jgarciab@ups.edu.ec ${ }^{2}$ \\ Verónica N. Espinoza \\ vespinoza@ups.edu.ec ${ }^{3}$
}

\section{Resumen}

En la actualidad, a nivel mundial, nacional, regional y local se presentan diferentes maneras de evadir impuestos, debido a que existen deficiencias en el control y fiscalización por parte de los gobiernos; sin duda, el elevado nivel de la evasión tributaria es algo que preocupa al Ecuador. Uno de los principales modus operandi para la evasión de impuestos ha sido la utilización de empresas fantasmas. Este trabajo presenta los resultados de una investigación con un enfoque mixto (cualitativo y cuantitativo) que toma como área de estudio una lista de 346 empresas fantasmas e inexistentes, publicadas en agosto de 2016 en la página del Servicio de Rentas Internas (SRI); los resultados muestran la caracterización de las diferentes empresas por su actividad, ubicación geográfica, constitución jurídica, aporte de capital, el perfil de los socios y sus empresas relacionadas. Los resultados obtenidos también permiten proponer estrategias de prevención y control que ayuden a disminuir la creación de empresas fantasmas.

Palabras clave: impuestos, evasión tributaria, empresas fantasmas, fiscalización.

1 Estudiante de último año de la Carrera de Contabilidad y Auditoría.

2 Doctorando en Proyectos de Desarrollo Rural y Gestión Sostenible de la Universidad Politécnica de Madrid Magíster en Administración de Empresas de la Universidad de Politécnica Salesiana, Master en Proyectos de Desarrollo Rural y Gestión Sostenible de la Universidad Politécnica de Madrid. Docente de la carrera de Contabilidad y Auditoría de la Universidad Politécnica Salesiana.

3 Doctorando en Contaduría en la Universidad Autónoma de Nuevo León. Magister en Auditoría Integral de la Universidad Técnica Particular de Loja, Docente de la Carrera de Contabilidad y Auditoría de la Universidad Politécnica Salesiana. 


\section{Abstract}

Nowadays, globally, nationally, regionally and locally, tax evasion is presented in different ways, due to the deficiencies in control and supervision by governments; No doubt the high level of tax evasion is something that has been worrying to Ecuador. One of the main modus operandi for tax evasion has been the use of ghost companies. This paper presents the results of a research with a mixed approach (qualitative and quantitative) that studies a list of 346 ghost and inexistent companies published in August of 2016 on the website of the Internal Revenue Service (SRI); The results show the characterization of the different companies by their activity, geographic location, legal constitution, investment capital, the profile of the partners and their related companies. The obtained results also allow proposing strategies of prevention and control that help diminishing the creation of ghost companies.

Key words: taxes, tax evasion, ghost companies, control. 


\section{Introducción}

De acuerdo a lo mencionado por Wettstein (1991), muchas empresas se han acostumbrado a la ilegalidad, llevando a cabo sus operaciones nacionales e internacionales a través de una compañía fantasma, de manera que no tienen que pagar impuestos en su país, por lo que el autor Sánchez (2001), expresa, que la evasión de impuestos constituye un proceso mediante el cual requiere la creación de planes estratégicos para implementar dicho proceso y así poder obtener ganancias con dinero ilícito.

Paredes (2015), indica que la Evasión Tributaria es un hecho que preocupa a todos los países del mundo, por los efectos que ha producido tales como: la disminución en los ingresos y déficit fiscal; es por eso que en una investigación realizada por la autora, se estima que los países en desarrollo pierden cada año 100.000 millones de dólares como consecuencia de la evasión de impuestos por parte de grandes empresas; igualmente Ramírez (2015), considera que las empresas fantasmas utilizan prácticas tributarias nocivas y que de esta manera los contribuyentes evitan pagar los respectivos valores por los impuestos que se encuentran establecidos en su país de domicilio.

Según Jorratt (1999), es importante considerar varios aspectos al momento de analizar la situación tributaria de cada una de las empresas, entre estos se mencionan a los siguientes: información acerca de la cuantía de la evasión tributaria, los mecanismos de evasión y los sectores económicos a los que pertenecen, esto permitiría a la Administración Tributaria orientar sus controles de fiscalización y modificarlos cuando sea necesario, por lo que es indispensable que se cuente con fiscalizadores experimentados y sistemas de información eficientes. Por otra parte, Villegas (2001), también ha definido que los impuestos son prestaciones en efectivo que el estado exige a sus ciudadanos por desarrollar una actividad económica en el país, es por eso que el propósito de estos es contar con recursos para atender múltiples demandas sociales y tratar de proveer bienestar a la sociedad.

Al respecto, la experiencia internacional de las distintas administraciones tributarias, aún las más adelantadas del mundo, indican que, en la actualidad, uno 
de los medios más utilizados para evadir impuestos es la creación de empresas fantasmas, por lo que la evasión fiscal es un tema de controversia global, en el cuál la lucha contra el fraude fiscal se ha convertido en una problemática. Berraza (2009).

Christensen (2015), director de $\mathrm{TJN}^{4}$, organización que lucha contra la evasión fiscal, explica que Estados Unidos, Brasil, Italia, Rusia y Alemania son países que cuentan con numerosas empresas dedicadas a la evasión de impuestos; en cuanto a Estados Unidos, el fisco estadounidense pierde aproximadamente $\$ 350.000$ millones anuales, a pesar de ser un país que tiene un alto índice de cumplimiento tributario, esta conducta es denominada "tax mórale"5 en la economía brasileña se ha verificado alrededor de \$280.000 millones de dólares anuales en evasión; lo que lo diferencia de Estados Unidos es que existe poca confianza en el Estado por lo que la gente, si puede, no paga impuestos; en Italia, su economía negra implica una pérdida de $\$ 238.000$ millones de dólares anuales y a pesar de que a partir del año 2007-2008 han existido cambios, muchos italianos no confían en el sistema; en Rusia, antes de la crisis el Banco Central reconocía que había una evasión fiscal de unos $\$ 35.000$ millones, el ingenio del evasor ruso son las "compañías de un día", que son las que no pagan impuestos ni a nivel municipal, regional o federal; en el año 2013 se estimó casi cuatro millones de este tipo de compañías; en cuanto a Alemania, el tamaño de su economía en negro representa un $16 \%$, lo que hace que ocupe el quinto lugar en el ranking de TJN.

A nivel de América Latina, Garguero (2015) distingue cuatro grupos de países en cuanto a cumplimiento tributario, por lo que expresa: "El primero está conformado por países que están relativamente bien en términos de cumplimiento tributario como Brasil, Argentina, Uruguay y Chile. Un segundo sería América Central, donde con alguna excepción, hay gigantescos niveles de informalidad y evasión. Un tercer grupo son países petroleros como México y Venezuela que se caracterizan por una pobre presión tributaria y mucho incumplimiento. $Y$ hay un cuarto grupo de países que viene mejorando mucho como Ecuador, Bolivia y Colombia".

4 La empresa TJN, cuyas siglas significan Tax Justice Network.

5 El significado en español de "tax mórale" es "ética impositiva". 
Al tratar de buscar una respuesta al ¿Por qué se realiza la evasión tributaria?, Hernando de Soto (1989), menciona que la excesiva interferencia gubernamental ocasiona que las empresas dediquen mucho tiempo a cumplir sus obligaciones con el estado; y Stanculescu (2014), formula que el exagerado incremento de impuestos genera insatisfacción en los contribuyentes, quienes molestos con las fuertes políticas fiscales establecidas por el Estado, terminan buscado la evasión. En este aspecto, los paraísos fiscales han jugado un papel fundamental, mediante la creación y uso de técnicas de evasión tributaria dentro de un sistema en el cual el propio Estado encubre y contempla varias posibilidades que permiten esquivar el control fiscal, en donde se permite la creación de empresas fantasmas o la apertura de cuentas bancarias sin tener la justificación del origen de los capitales que reposan en ellas.

En cuanto a Ecuador, una investigación realizada por Castro (2015), manifiesta que el gobierno ha efectuado algunos cambios tributarios en estos últimos años, considerando como principio fundamental, la igualdad. Además, busca mantener un presupuesto equilibrado, razón por la cual requiere disminuir desequilibrios, aumentar ingresos fiscales y combatir la evasión tributaria.

Por otra parte Arias (2010), expresa que el Ecuador ha enfrentado varios problemas relacionados con la evasión y elusión de impuestos, como son: la constitución de empresas fantasmas, la falsa creación de gasto o crédito tributario, la sobrevaloración o subvaloración de transacciones internacionales y la ausencia de normas relativas a la fiscalidad del comercio electrónico, debido a que el gobierno le ha dado una mayor importancia a la existencia de mayores recursos petroleros que facilitan el financiamiento del presupuesto general del Estado, haciendo que la sociedad le quite importancia a los ingresos tributarios y esto permita que varias reformas tributarias no sean aprobadas por el pueblo ecuatoriano. Por el contrario, la política fiscal se ha dedicado a proponer nuevas exoneraciones tributarias y esto es lo que produce una inequidad dentro del sistema tributario ecuatoriano y limitaciones en el control de la Administración Tributaria. 
$\mathrm{EI} \mathrm{SRI}{ }^{6}$ de Ecuador, mostró como resultado que las empresas fantasmas ingresan dentro del $\mathrm{RUC}^{7}$, direcciones en las cuáles presuntamente se realizan actividades económicas por las cuales fueron constituidas. Sin embargo, luego de la revisión efectuada por el SRI, se verificaron lugares inexistentes y varios casos donde los inmuebles no eran adecuados para la realización de grandes operaciones comerciales. (SRI, 2016).

En consecuencia, el Superintendente de Bancos Pedro Solines (2014), en una entrevista realizada por Diario El Comercio señala que la problemática de las empresas fantasmas se agravó en Ecuador durante los últimos dos años, debido a que las instituciones representadas por personas naturales o jurídicas que ejercen actividades de intermediación financiera proceden de manera irregular, incurriendo en lavado de activos, estafas, uso doloso de plataformas electrónicas y otros actos ilícitos. Teniendo en cuenta estos antecedentes, este artículo plantea responder a las siguientes preguntas de investigación: ¿Qué características tienen las empresas fantasmas en Ecuador?, ¿Qué perfil tienen los socios que constituyen empresas fantasmas? y ¿Cuáles son las empresas con las que se relacionan?; al mismo tiempo, se proponen estrategias de prevención y control ante la creación de este tipo de empresas.

\section{Metodología}

La primera parte de esta investigación fue el análisis de la problemática de la evasión tributaria que se ha provocado por las empresas fantasmas en el mundo y que ha generado interés en la administración tributaria ecuatoriana en los últimos años. El artículo académico se realizó bajo un enfoque mixto (cualitativo y cuantitativo). Con respecto al enfoque cualitativo se realizó un análisis de cada uno de los socios relacionados con las empresas fantasmas; y en el enfoque cuantitativo se determinó el porcentaje con el que cuenta cada región del Ecuador en cuanto a dichas compañías.

La elaboración del presente artículo se realizó en base al análisis de la lista de 346 empresas fantasmas e inexistentes publicadas en agosto 2016 en la página del Servicio de Rentas Internas del Ecuador, sin tomar en cuenta una muestra.

6 La institución SRI, cuyas siglas significan Servicio de Rentas Internas

7 El significado de las siglas RUC es: Registro Único de Contribuyentes 
A través de la página de la Superintendencia de Compañías fue posible conocer las fechas de constitución, la nacionalidad, en qué provincia, cantón y ciudad se encuentran ubicadas, si es o no proveedora de bienes o servicios del estado, la actividad de la empresa, el tipo de compañía de acuerdo a su constitución, el capital social, los socios y su relación con otras compañías. Posteriormente en la página de la SENESCYT ${ }^{8}$ con el número de cédula de los socios se verificó si contaban con título de tercer o cuarto nivel.

Y finalmente, para presentar los resultados obtenidos en el presente artículo, se utilizaron gráficos con el fin de demostrar de manera más explícita la incidencia que han tenido las empresas fantasmas en cada una de las regiones del Ecuador.

De igual manera, cabe mencionar también, que la presente investigación es de tipo documental-descriptiva, puesto que se determinan las principales características del fenómeno analizado, a efecto de recolectar información para así describir lo que se investiga; y al mismo tiempo constituye una línea con un valioso potencial de desarrollo investigativo y científico, al no existir investigaciones de mayor impacto sobre el tema.

\section{Resultados}

\subsection{Resolución Impugnada}

Luego de analizar las 346 empresas fantasmas e inexistentes publicadas por el SRI, se pudo observar que 317 empresas cuentan con una resolución negativa, es decir, que estas compañías no han logrado cumplir con sus obligaciones tributarias; mientras que 21 se encuentran en trámite y 8 se ratifican. Es por ello que se procedió a analizar a las 317 empresas que no cuentan con una resolución.

8 SENESCYT: Secretaría de Educación Superior, Ciencia, Tecnología e Innovación 
Gráfico 1. Resolución Impugnada

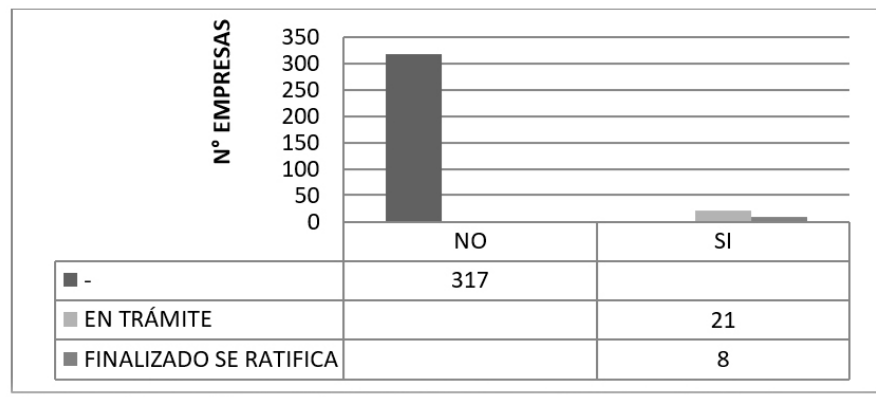

Fuente: elaboración propia

\subsection{Ubicación geográfica}

En el siguiente gráfico se encuentra la ubicación que tienen las diferentes empresas fantasmas en el Ecuador; 2 se ubican en la provincia del Azuay, 2 en El Oro, 279 en el Guayas, 4 en Manabí, 22 en Pichincha, 1 en Santo Domingo de los Tsáchilas y 7 compañías se encuentran liquidadas, dando el total de 317 empresas analizadas.

Gráfico 2. Empresas por Provincia

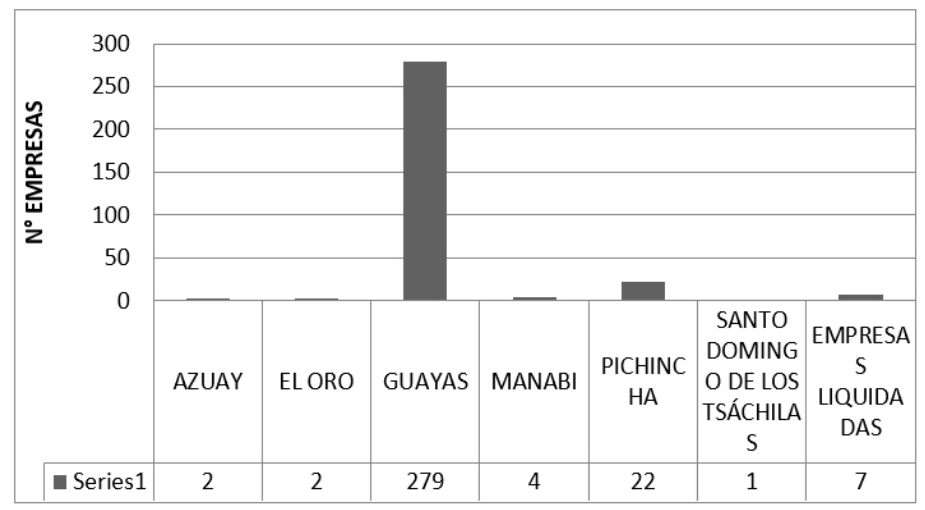

Fuente: elaboración propia 


\subsection{Tipo de compañías}

Las empresas fantasmas en su mayoría son Sociedades Anónimas, teniendo un total de 288: 10 de Responsabilidad Limitada, 1 Sucursal extranjera, 11 unipersonales y 7 compañías se encuentran liquidadas.

Gráfico 3. Tipo de Compañías

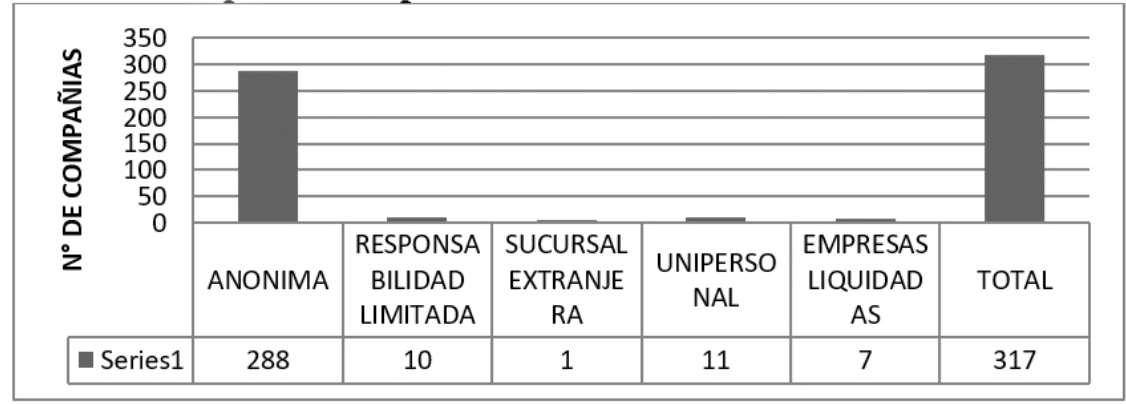

Fuente: elaboración propia

\subsection{Actividad económica}

De acuerdo a la Clasificación Nacional de Actividades Económicas CIIU existen varias actividades económicas en las Sociedades Anónimas, pero entre las actividades predominantes de las 317 empresas se tienen las siguientes: el Comercio al por mayor, excepto vehículos con un $43 \%$, seguido por Actividades Inmobiliarias con el 14\%, Construcción de edificios 13\%, Comercio al por menor, excepto vehículo con el $9 \%$ y en menor medida otras actividades como Consultoría de Gestión y Publicidad 5\%, Obras de Ingeniería Civil 4\%, Agricultura y Jurídicas-Contabilidad 3\%. 
Gráfico 4. Actividad Económica (SOCIEDAD ANÓNIMA)

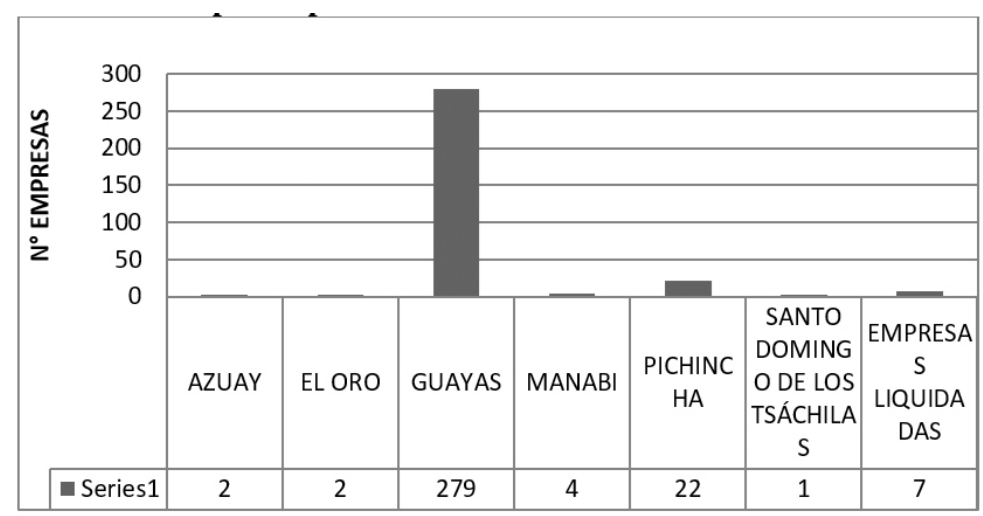

Fuente: elaboración propia

Respecto a las Empresas de Responsabilidad Limitada, las actividades predominantes de las 317 empresas son: Jurídicas-Contabilidad y Agencias de viajes alcanzando el $20 \%$, seguido por otras actividades como Seguridad, Inmobiliarias, Comercio al por mayor, excepto vehículos, construcción de edificios, fabricación de equipo eléctrico y transporte por vía acuática conformando el $10 \%$.

Gráfico 5. Actividad Económica (CIAS. RESPONSABILIDAD LIMITADA)

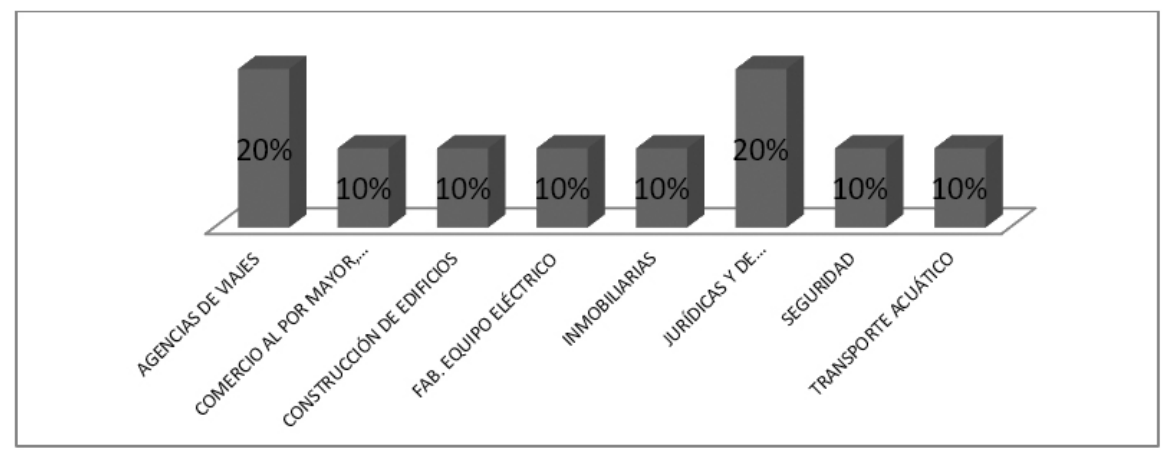

Fuente: elaboración propia 


\subsection{Aporte de capital}

Un $79 \%$ de las Sociedades Anónimas se forman con un capital de $\$ 800$, seguido por el 16\% entre $\$ 801$ a $\$ 1000$, y en menor medida por el $2 \%$ entre $\$ 1001$ a $\$ 5000$; y el 3\% entre $\$ 5001$ a $\$ 50.000$.

Gráfico 6. Aporte de Capital (SOCIEDAD ANÓNIMA)

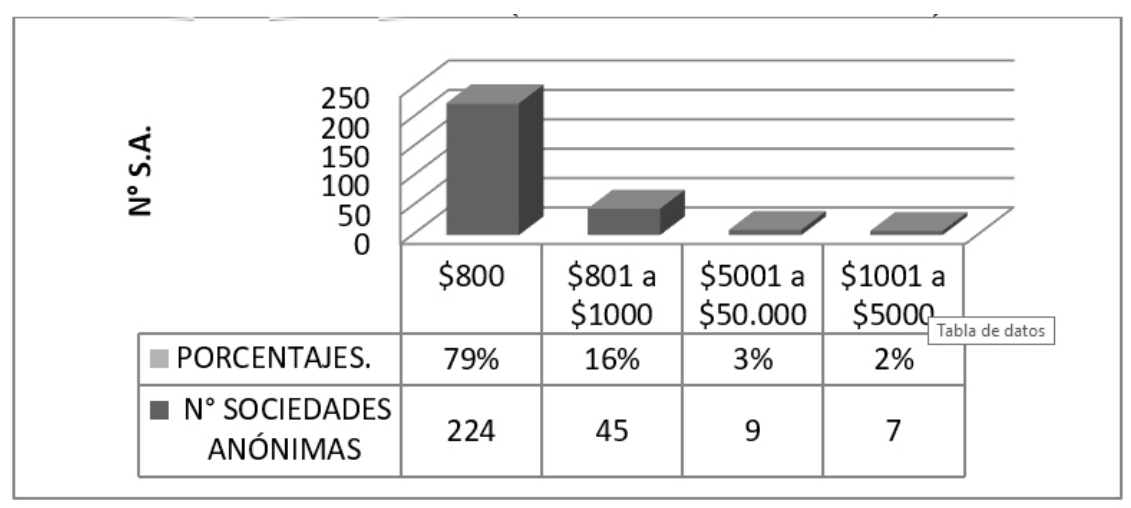

Fuente: elaboración propia

El $50 \%$ de las empresas de Responsabilidad Limitada se conforman con $\$ 400$, seguido por el $20 \%$ con $\$ 1000$, y el $10 \%$ con $\$ 750, \$ 10000$ y $\$ 15000$ respectivamente.

Gráfico 7. Aporte de Capital (CIAS. RESPONSABILIDAD LIMITADA)

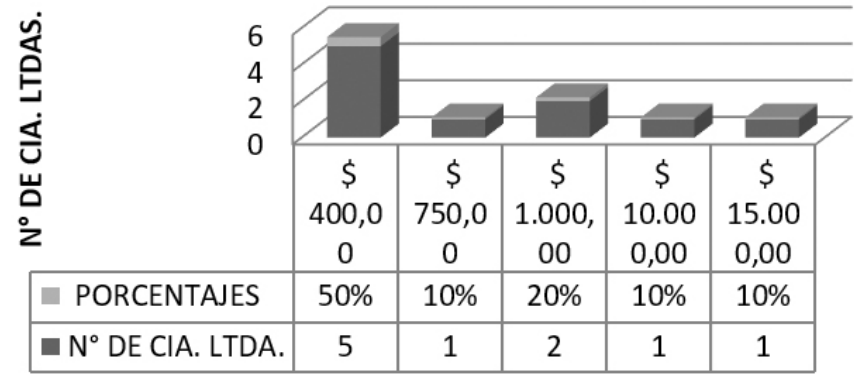

Fuente: elaboración propia 


\subsection{Perfil de los socios (Empresas Relacionadas)}

El 79\% de las empresas fantasmas no tienen compañías relacionadas, mientras que el $21 \%$ sí lo tienen, es decir varios socios mantienen relación con otras empresas ecuatorianas.

Gráfico 8. Empresas Relacionadas

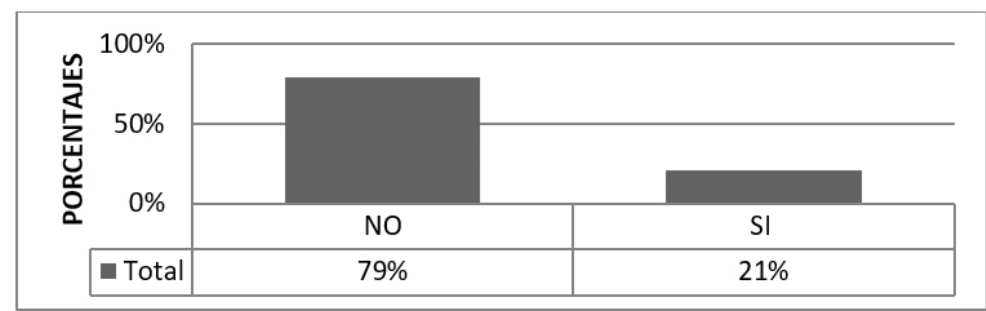

Fuente: elaboración propia

Existen socios de las diferentes empresas fantasmas que se encuentran relacionados con otras, en el Gráfico 9 se puede observar que 32 socios están vinculados con 1 empresa, 16 socios con 2, 11 socios con 3,4 socios con 4,3 socios con 5 y por último 1 socio con 7 empresas.

Gráfico 9. $\mathrm{N}^{\circ}$ de socios relacionados con otras empresas.

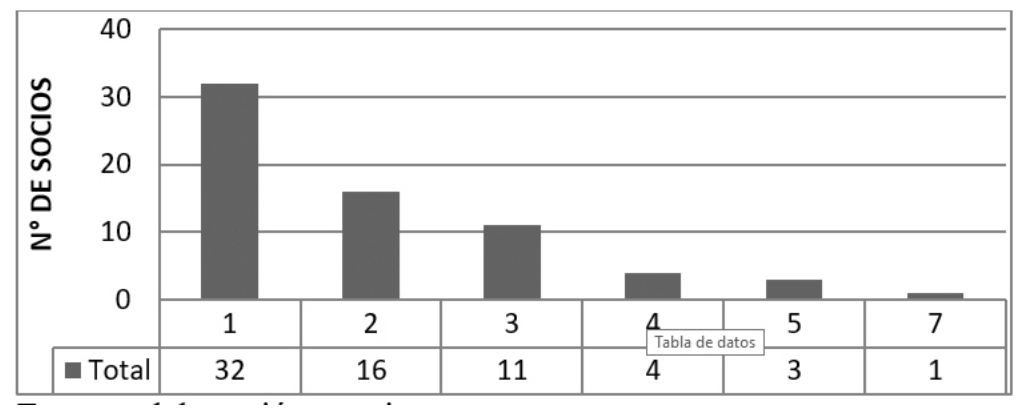

Fuente: elaboración propia 
Los resultados que se presentan en el siguiente gráfico indica que 19 empresas cuentan con 2 socios, 2 empresas con 21 socios y en menor medida con 4, 5,6 y 9 socios. Cabe señalar que existen socios de nacionalidad extranjera, estos son residentes de: Perú, Estados Unidos, Argentina, Uruguay, Colombia, España, Cuba, Rusia, Brasil, Chile, Venezuela y Corea del Sur.

Gráfico 10. № de empresas relacionadas con distintos socios.

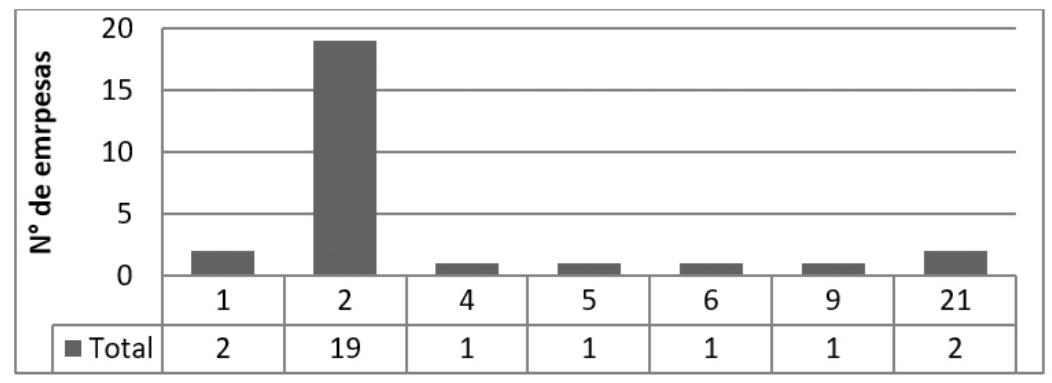

Fuente: elaboración propia

\subsection{Perfil de los socios (nivel de instrucción formal)}

Respecto al nivel de instrucción formal, se procedió a verificar en la página de la SENESCYT si cuentan con algún título, el 63\% de los socios no cuentan, mientras que el $37 \%$ si cuentan con una formación de tercer y cuarto nivel.

Gráfico 11. Nivel de instrucción formal universitaria de los socios

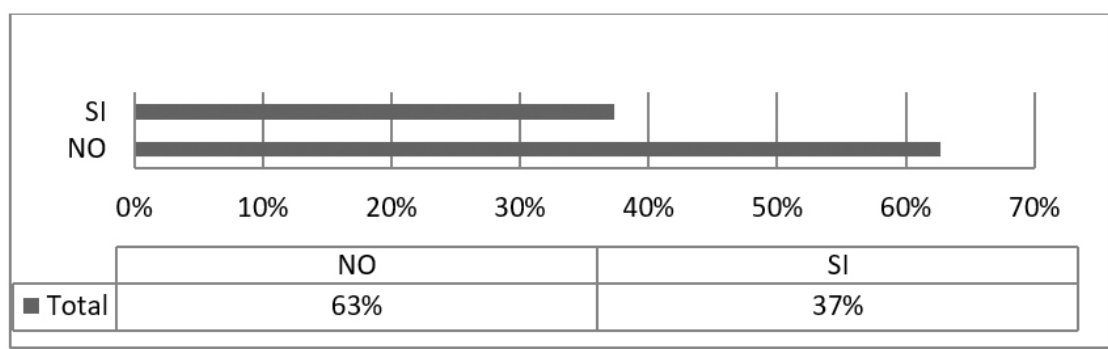

Fuente: elaboración propia. SENESCYT (Secretaría de Educación Superior, Ciencia, Tecnología e Innovación). 


\section{Discusión y Conclusión}

Mediante la investigación que se realizó sobre las empresas fantasmas e inexistentes en el Ecuador se pudo determinar que aún existe un número considerable de las mismas, a pesar de que el Servicio de Rentas Internas se encuentra realizando una investigación exhaustiva con la finalidad de reducir estas prácticas de planificación agresiva y de esta manera asegurar el correcto cumplimiento de las obligaciones tributarias para el desarrollo del país.

A lo largo de la investigación, se pudo determinar que existe una escasez de publicaciones sobre empresas fantasmas mundiales; pero se realizó una comparación entre el Ecuador y diferentes países como Estados Unidos y Rusia. En una publicación realizada por Diario El Telégrafo (2014) se dice que el Ecuador pierde entre $\$ 300$ y $\$ 400$ millones de dólares anuales por evasión fiscal, partiendo de que siempre se ha generado una resistencia al momento de cancelar los respectivos valores por concepto de impuestos. El alto índice de evasión de impuestos es un tema que aún preocupa a los directivos del Servicio de Rentas Internas, de tal forma que Miguel Avilés (Director Regional del SRI 2011) indicó que al cierre del año 2011 este delito bordeó los 4 mil millones de dólares; el Banco Mundial indica asimismo que el dinero que se oculta de forma deliberada para evitar el pago de impuestos en el mundo es de más del 18\% del PIB global; al mismo tiempo Taxi Justice Network (TJN) calcula que la evasión de impuestos en el mundo representa aproximadamente 3 billones de dólares, provocando con ello que sólo existan recursos para cubrir las necesidades básicas de una población y que no exista desarrollo en el país. Con respecto a Estados Unidos, más de 300.000 millones de dólares corresponden a la evasión de impuestos; la razón principal por la que el fisco estadounidense deja de cobrar estos tributos es porque la normativa de este país es muy flexible. De igual manera la evasión fiscal es un negocio en Rusia, según un estudio, hasta el $60 \%$ de las empresas rusas utilizan empresas tapadera que se crean de forma temporal para ocultar activos y evitar pagar impuestos. (Keating, mdzol.com, 2014).

Con respecto a Ecuador, de acuerdo a la Clasificación Nacional de Actividades Económicas CIIU la mayoría de las sociedades anónimas se dedican al comer- 
cio al por mayor, excepto vehículos alcanzando el $43 \%$, seguido por actividades inmobiliarias con el 14\%, construcción de edificios $13 \%$ y en menor medida actividades de comercio al por menor, excepto vehículos, consultoría de gestión, publicidad, obras de ingeniería civil, agricultura y jurídicas-contabilidad; mientras que las empresas de responsabilidad limitada las actividades jurídicas-contabilidad y agencias de viajes representan el $20 \%$ y el $10 \%$ están conformadas por otras actividades. Los resultados permiten identificar la fuerte presencia de empresas fantasmas en la provincia del Guayas, pero también existen en EI Oro, Manabí y Santo Domingo de los Tsáchilas, es decir, se encuentran en su mayoría en la Costa; región en donde debería existir un control más riguroso ya que es donde los socios constituyen estas compañías para poder realizar sus actividades fantasmas y así evitar pagar impuestos al fisco. En cuanto al tipo de compañías existe un índice alto de Sociedades Anónimas y en menor medida también se encuentran empresas de Responsabilidad Limitada y Unipersonales que están conformadas en porcentajes mínimos pero que existen en la base de datos de las 346 empresas fantasmas. De estas evidencias, la mayoría son sociedades anónimas porque para su constitución se necesitan mínimo de dos socios, sin tener en cuenta un máximo, por ende esto significa para estas compañías una ventaja, porque pueden ingresar un número ilimitado de personas para conformar una empresa fantasma, generando problemas para la administración tributaria porque no se tiene ni idea de quienes son los dueños, así que no se sabe a quién hay que cobrar los impuestos.

Por otro lado, un factor de significativa importancia en las empresas fantasmas está relacionado con el tema de los socios que conforman las mismas, los resultados del estudio demuestran que existen socios de diferentes nacionalidades como: Brasil, Chile, Cuba, Venezuela, Argentina, Uruguay, Colombia, Corea del Sur y Estados Unidos. Cabe señalar que existe una empresa de nacionalidad española, pero que, la misma desarrolla sus actividades en la ciudad de Quito. En cuanto al número de socios que conforman estas empresas fantasmas, en su mayoría están constituidas por 2 socios, pero existen dos empresas que tienen 21 socios.

La creación de empresas fantasmas en el Ecuador frena la recaudación tributaria en el país, ocasionando problemas para la administración tributaria, lo que 
provoca una disminución en la recaudación de tributos. Los impuestos constituyen el sustento básico del presupuesto gubernamental, de tal manera que al no cumplir con esta obligación se genera un déficit fiscal, lo que origina que, para cubrirlo, se creen nuevos tributos con el fin de compensar lo que no se logró recaudar por estas compañías.

Un estado con baja capacidad de recaudación, se lo denomina frágil porque impide un financiamiento sostenible de bienes públicos y de protección social, con lo cual las decisiones pueden resultar poco efectivas. Asimismo en una entrevista realizada al Departamento Jurídico de la Superintendencia de Compañías, se menciona que la creación de empresas fantasmas en el Ecuador ocasiona problemas para el país porque se desmotiva la actividad productiva; y en tal virtud las mismas no generan producción ni tributos al Estado; también se señala que la normativa tributaria actualmente se ha mejorado a través de varias reformas que son las que controlan al sector de la construcción inmobiliaria y automotor, donde existían gran parte de estas compañías denominadas fantasmas.

En el Ecuador el capital mínimo para conformar una sociedad anónima es de $\$ 800$ y para una empresa de responsabilidad limitada es de $\$ 400$, estos valores en comparación con otros países no son muy representativos, dando paso a crear con mayor facilidad una empresa fantasma; con respecto a Uruguay el monto en dólares para constituir una sociedad anónima es de \$3.200 y una sociedad limitada es de \$750; mientras que en Argentina es de \$628,10 para una sociedad de responsabilidad limitada y $\$ 6.281$ para una sociedad anónima, por otra parte en España el capital para una sociedad limitada es de 3000 euros (\$3210) y una sociedad anónima 60.000 euros (\$64.199); al comparar estos valores se determinó que en el país el capital para constituir una compañía es menor con respecto a Uruguay, Argentina y España. Luego de realizar una revisión bibliográfica de estos países, se encontró que para constituir una empresa en Argentina se necesitan de 25 días hábiles, la mayoría de trámites se realizan personalmente y el sistema tributario y administrativo es burocráticamente estricto; mientras que en una publicación realizada por Diario El Telégrafo (2014), se dice que en el Ecuador ya se pueden constituir empresas en 2 días, los gastos por constitución disminuyen de $\$ 3000$ a $\$ 200$ y los trámites se realizan por la web. Por otra parte, Diario El Mundo (2012) señala que, los mejores países para 
emprender son: Nueva Zelanda que permite crear una empresa con un trámite $100 \%$ online y con un pago único de 100 euros (\$107), Australia con un procedimiento de apenas 2 días se crea una empresa y un pago de 300 euros (\$321), Canadá no requiere de un capital mínimo y Estados Unidos donde aproximadamente en 6 días y $\$ 535$ cualquiera puede emprender su empresa.

Por último, se proponen estrategias de prevención y control ante la creación de empresas fantasmas, las mismas que su aplicación o puesta en marcha dependen de agentes externos, en este caso sería el Estado:

1. Establecer mayores controles en las provincias donde se encuentran la mayoría de las empresas fantasmas, por ejemplo: verificar si estas compañías se encuentran ubicadas en la misma provincia o si se procedió a cambiar su residencia. La administración tributaria deberá operar a través de intervenciones rápidas, mediante técnicas de auditoría fiscal.

2. Modificar el capital para la constitución de sociedades tanto anónimas como limitadas.

3. Conocer a breves rasgos información de los socios que conforman una compañía en el Ecuador, es decir, percatarse de qué tipo de personas forman parte de la misma, número de empresas donde es socio, si cumple con sus obligaciones tributarias; esta estrategia permitiría identificar a la persona, por lo que si se encuentra algo extraño se proceda a realizar un seguimiento.

4. El Estado debería incrementar su rol de educador en el tema de tributos, con el fin de informar a la ciudadanía sobre los efectos positivos del pago de los mismos y la nocividad que produce la omisión del ingreso de aquellos.

5. Revisar cada cierto tiempo la documentación presentada por los representantes legales de las compañías, para cerciorarse de que cualquier cambio efectuado por los mismos se haya informado a la institución competente o se ha desarrollado la actualización correspondiente.

6. Efectuar una campaña de publicidad más agresiva por parte de los medios de comunicación nacional, con la finalidad de otorgar un mayor conocimiento a la sociedad en general sobre estos delitos, como es la creación de las empresas fantasmas.

7. Los profesionales relacionados con el tema contable de una empresa, como por ejemplo: los contadores públicos, deberían canalizar y reformar medi- 
das de prevención para reconocer a estas compañías y en el momento de detectar alguna anomalía sean los primeros en denunciar, con la finalidad de ayudar al país en general y salvar su integridad como profesional.

8. Mantener un constante y permanente intercambio de información, ya que el SRI de nuestro país necesita información abierta y disponible de los países con los cuales se realizan operaciones económicas, con el fin de poder detectar algún acto ilícito en el ámbito fiscal mediante el análisis de todas las transacciones que se realicen entre estos países.

9. Sería importante para el Ecuador implementar un convenio con otros países con el fin de intercambiar información de cada una de las empresas domiciliadas en el respectivo país. 


\section{Referencias bibliográficas}

- Arias, S. (2010). Los Paraísos Fiscales y su Incidencia en el ámbito Tributario Ecuatoriano, 212, 12-20.

- Castro, L. (2015). Análisis de las reformas tributarias e incidencia en la recaudación de los principales impuestos del Ecuador 2009-2013, 109, 12 15.

- Chris tensen, J. (2015). Los campeones de la evasión fiscal en el mundo. Recuperado el 25 de noviembre de 2016, disponible en: http://www.bbc. com/mundo/noticias/2015/04/150406_economia_evasion_fiscal_america_ latina_mj

- Diario El Comercio (2014). La aparición de empresas fantasmas en el sector financiero de Ecuador se agudizó en los dos últimos años. Recuperado el 10 de enero de 2017, disponible en: http://www.elcomercio.com/actualidad/ negocios/aparicion-de-empresas-fantasmas-sector.html

- Diario El Mundo (2012). Los diez mejores países para emprender. Recuperado el 11 de enero de 2017, disponible en: http://www.elmundo. es/blogs/elmundo/hay-un-guru-en-mi-sopa/2012/10/04/los-10-mejorespaises-para-emprender.html

- Diario El Telégrafo (2014). En Ecuador ya se pueden constituir empresas en dos días. Recuperado el 15 de enero del 2017, disponible en: http:// www.eltelegrafo.com.ec/noticias/economia/8/en-ecuador-ya-se-puedenconstituir-empresas-en-2-dias

- Garguero, J. (2015). Los campeones de la evasión fiscal en el mundo. Recuperado el 25 de noviembre de 2016, disponible en: http://www.bbc. com/mundo/noticias/2015/04/150406_economia_evasion_fiscal_america_ latina_mj

- Jorratt, M. (1999). Estimación de la evasión tributaria en Chile, 39, 4-17.

- Paredes, P. (2015). La evasión tributaria e incidencia en la recaudación del impuesto a la renta de personas naturales en la provincia del Guayas, período 2009-2012, 151, 20-22.

- Ramírez, C. (2015). Los paraísos fiscales y las prácticas tributarias nocivas que afectan la administración tributaria en el Ecuador, 108, 2,5. 
- Sánchez, L. (2001). Lavado de dinero: un delito transnacional (pp.2-3). Buenos Aires.

- Secretaría de Educación Superior, Ciencia, Tecnología e Innovación. Consulta de títulos. Recuperado el 22 de diciembre de 2016, disponible en: http://www.senescyt.gob.ec/consulta-titulos-web/faces/vista/consulta/ consulta.xhtml

- Servicio de Renta Internas (2016). Listado de Empresas Fantasmas e Inexistentes. Recuperado el 20 de noviembre de 2016, disponible en: http:// www.sri.gob.ec/web/guest/empresas-inexistentes

- Soto, H. (1989). El otro sendero, 15, 4-12.

- Stanculescu, E. (2014). Análisis Jurídico de los Paraísos Fiscales: Medios de Evasión o de Elusión Fiscal, 143, 3-5.

- Superintendencia de Bancos (2016). Portal de usuario. Recuperado el 25 de noviembre de 2016, disponible en: http://portaldelusuario.sbs.gob.ec/ contenido.php?id_contenido $=66$

- Villegas, H. (2001). Curso de Finanzas, Derecho Financiero y Tributario. Buenos Aires: JJCPM.

- Wettstein, G. (1991). Los impunes, una minoría peligrosa. Nueva Sociedad,111, 158-167. 